\title{
Structure of the core of magnetic vortices in $d$-wave superconductors with a subdominant triplet pairing mechanism
}

\author{
Mikael Fogelström \\ Department of Microtechnology and Nanoscience, S-41296 Göteborg, Sweden
}

(Received 5 August 2011; published 26 August 2011)

\begin{abstract}
The quasiparticle states found in the vortex core of a high- $T_{\mathrm{c}}$ cuprate superconductor may be probed by scanning tunneling spectroscopy. Results of such experiments have revealed typical spectra that are quite different from what is seen in conventional low- $T_{\mathrm{c}}$ superconductors. In particular, the Caroli-de Gennes-Matricon state at $E \sim 0$ in the core center is not seen. Instead, in a high- $T_{\mathrm{c}}$ vortex core, quasiparticle states are found at energies that are at a sizable fraction of the gap energy. One explanation for this could be that a finite amplitude of a competing order parameter stabilizes in the vortex-core center. Here I will explore the possibility of nucleating a vortex-core state that locally breaks inversion symmetry. The vortex-core order parameter is of mixed parity, a $[d+i p]$ wave, and the quasiparticle spectra in the core center lacks the $E=0$ states.
\end{abstract}

DOI: 10.1103/PhysRevB.84.064530

PACS number(s): 74.25.Ha, 74.50.+r, 74.55.+v

\section{INTRODUCTION}

In conventional, nominally clean, type-II superconductors, the quasiparticle spectrum in a vortex core was described by Caroli, de Gennes, and Matricon. ${ }^{1}$ They found that quasiparticle states are localized in the vortex core, and that these same states carry the currents that screen the magnetic flux line from penetrating the interior of the superconductor. A direct measurement of vortex-core states was done by scanning tunneling spectroscopy (STS) ${ }^{2}$ and the measured data could be quantitatively explained by theory. ${ }^{3}$ Performing STS measurements on vortices in high- $T_{\mathrm{c}}$ cuprates, on yttrium barium copper oxide $(\mathrm{YBCO})^{4}$ or on bismuth strontium calcium copper oxide $(\mathrm{BSCCO})^{5-7}$ (see also references in Ref. 8) revealed a very different generic picture; the vortex core in a high- $T_{\mathrm{c}}$ superconductor does not harbor pronounced core states. By theory ${ }^{9-12}$ these core states should be present also for an order parameter of $d$-wave symmetry relevant for high- $T_{\mathrm{c}}$ superconductors and thus readily be seen in STS. Instead, nondispersing vortex states are seen at energies corresponding to $\sim 30 \%$ of the bulk value of the superconducting energy gap $\Delta_{0}$.

Theoretical suggestions to explain the vortex-core spectra seen in high- $T_{\mathrm{c}}$ cuprates include possible subdominant singletparing amplitudes, ${ }^{11-13}$ antiferromagnetic order stabilizing in the vortex core ${ }^{14,15}$ the normal-state pseudogap phase made visible in the core, ${ }^{16}$ or competition between $d$-density wave and $d$-wave superconducting order. ${ }^{17}$ In this paper I will explore the possibility of nucleating a vortex-core state of mixed parity and show that this state may have a finite amplitude of a $p$-wave order parameter in the vortex center. The possibility of a mixed singlet-triplet pairing state in a vortex core has been suggested to occur in an $s$-wave superconductor as a result of spin-orbit coupling. ${ }^{18}$ In the context of $d$-wave superconductors both spin-orbit coupling and the presence of a Zeeman coupling has been considered, finding a singlet-triplet mixing in the vortex phase in the Ginzburg-Landau region $\left(0 \ll T \lesssim T_{\mathrm{c}}\right) \cdot{ }^{19-21}$ Here, I will use the microscopic quasiclassical theory to show, given an attractive pairing interaction in a $p$-wave channel and a weak intrinsic Zeeman coupling to the magnetic field to break the spin-singlet symmetry of the parent $d$-wave supeconductor, that a sizable $p$-wave order parameter may stabilize locally in the $d$-wave vortex-core region. Using this self-consistently determined order-parameter field, I then compute the spatially resolved local density of states in the vortex. It turns out that the quasiparticle spectra seen in the STS on the high- $T_{\mathrm{c}}$ cuprates $^{4-8}$ can, to a large extent, be reproduced theoretically as a direct consequence of this triplet superconducting core order.

The scenario is that a finite amplitude of a competing order parameter stabilizes in the vortex core. An isolated singly quantized vortex has the asymptotic order parameter $\Delta(\boldsymbol{R})=\Delta_{\infty} e^{i \phi}$ as one circles the core. The phase winding of $2 \pi$ corresponds to a center-of-mass angular momentum $\hat{L}_{z}^{\text {c.m. }} \Delta(\boldsymbol{R})=\frac{\hbar}{i} \frac{\partial}{\partial \phi} \Delta(\boldsymbol{R})=\hbar \Delta(\boldsymbol{R})$ of the Cooper pairs. Approaching the vortex core, the order-parameter amplitude is gradually suppressed and vanishes in the origin of the vortex so as to maintain the single valuedness of $\Delta(\boldsymbol{R})$. To have a finite order-parameter amplitude in the core, the center-of-mass angular momentum can rotate in to an internal orbital angular momentum of the Cooper pair $\hat{L}_{z}^{\text {orb }} \Delta(\boldsymbol{p}, \boldsymbol{R})=\frac{\hbar}{i} \frac{\partial}{\partial \phi_{\hat{\mathrm{p}}}} \Delta(\boldsymbol{p}, \boldsymbol{R})=$ $\hbar \Delta(\boldsymbol{p}, \boldsymbol{R}){ }^{22}$ This scenario occurs in the $B$ phase of superfluid ${ }^{3} \mathrm{He}$ where the $A$ phase and double-core states are found to be energetically favorable to a normal-state core in different regions of the pressure-temperature phase diagram. ${ }^{18,23-26}$

A $d$-wave superconductor has singlet-pairing symmetry, and to have a finite order parameter in the core with $L_{z}^{\text {orb }}=\hbar$, a $p$-wave order parameter is needed in the vortex-core region. This requires (i) an attractive pairing interaction in a triplet channel, and (ii) a symmetry-breaking field that introduces a seed of a triplet component. The first condition I argue to be intrinsic in a spin-fluctuation-mediated pairing relevant for high- $T_{\mathrm{c}}$ superconductors (see Ref. 27 and references therein). This interaction can support condensation into both spinsinglet and spin-triplet superconducting states. ${ }^{28}$ The second condition is readily given by the weak Zeeman coupling to an external field that is always present in a vortex. This is in particular true for extreme type-II superconductors where the penetration depth is far larger that the coherence length. In this paper I neglect orbital effects and the screening of the external 
magnetic field and assume that the external magnetic field is constant over the vortex-core region.

\section{MODEL}

For highly anisotropic spin fluctuations $\chi^{z} \equiv \chi_{z z} \gg$ $\chi_{x x, y y} \equiv \chi^{\perp}$, a susceptibility that can be tuned from predominantly antiferromagnetic $(\delta \approx 0)$ to ferromagnetic $(\delta \approx \pi)$ spin fluctuations can be modeled as

$$
\chi^{z}(\boldsymbol{q})=\sum_{\delta_{x, y}= \pm \delta} \frac{\chi_{Q} / 4}{1+4 \xi_{\mathrm{sfl}}^{2}\left(\cos ^{2} \frac{q_{x}-\delta_{x}}{2}+\cos ^{2} \frac{q_{y}-\delta_{y}}{2}\right)} .
$$

$\chi_{Q}$ is the overall amplitude and $\xi_{\text {sfl }}$ is the spin-spin correlation length which is typically a few lattice constants $(a)$ in the cuprates. Introducing a simple coupling $g$ between the spin fluctuations and the quasiparticles, the pairing interaction is $V\left(\boldsymbol{p}-\boldsymbol{p}^{\prime}\right) \equiv V(\boldsymbol{q})=\mathcal{N}_{f} g^{2} \chi^{z}(\boldsymbol{q})=\bar{\chi}^{z}(\boldsymbol{q}), \mathcal{N}_{f}$ being the total density of states at the Fermi level. The resulting gap equation allows for three channels of pairing, one spin singlet with $V_{s}(\boldsymbol{q})=\bar{\chi}^{z}(\boldsymbol{q}) / 2$ and two spin-triplet channels, one with $V_{t z}(\boldsymbol{q})=\bar{\chi}^{z}(\boldsymbol{q}) / 2$ having the $\boldsymbol{d}$ vector parallel to $\hat{z}$ and one $V_{t \perp}(\boldsymbol{q})=-\bar{\chi}^{z}(\boldsymbol{q}) / 2$ for which $\boldsymbol{d} \perp \hat{z}$. Using Eq. (1) the gap equation in a weak-coupling approximation reads

$$
\Delta_{x}\left(\boldsymbol{p}_{F}\right)=-T \sum_{\left|\epsilon_{n}\right| \leqslant \epsilon_{c}}\left\langle V_{x}\left(\boldsymbol{p}_{F}-\boldsymbol{p}_{F}^{\prime}\right) n\left(\boldsymbol{p}_{F}^{\prime}\right) f_{x}\left(\boldsymbol{p}_{F}^{\prime} ; \epsilon_{n}\right)\right\rangle \boldsymbol{p}_{F}^{\prime} \text {. }
$$

Here $f_{x}\left(\boldsymbol{p}_{F} ; \epsilon_{n}\right)(x=s, t z, t \perp)$ is the anomalous propagator at Matsubara frequency $\epsilon_{n}$ and momentum $\boldsymbol{p}_{F}$ and $n\left(\boldsymbol{p}_{F}\right)=\left|\boldsymbol{v}_{F}\left(\boldsymbol{p}_{F}\right)\right|^{-1} /\left\langle\left|\boldsymbol{v}_{F}\left(\boldsymbol{p}_{F}^{\prime}\right)\right|^{-1}\right\rangle \boldsymbol{p}_{F}^{\prime}$. I use a linearized version of Eq. (2), assuming the factorization $V_{x}\left(\boldsymbol{p}_{F}-\right.$ $\left.\boldsymbol{p}_{F}^{\prime}\right)=\sum_{\Gamma} \lambda_{\Gamma} \mathcal{Y}_{\Gamma}\left(\boldsymbol{p}_{F}\right) \mathcal{Y}_{\Gamma}^{*}\left(\boldsymbol{p}_{F}^{\prime}\right)$, together with a tight-binding parametrization of the band structure relevant for BSCCO ${ }^{29}$ to compute the eigenvalue spectra for the possible pairing symmetries as a function of $\delta$, keeping $\xi_{\text {sfl }}=2 a$. To each eigenvalue $\lambda_{\Gamma}$ belongs a set of basis functions $\mathcal{Y}_{\Gamma}\left(\boldsymbol{p}_{F}\right)$ which may be classified according to the irreducible representations $(\Gamma)$ of the crystal group $D_{4 h}$. The resulting eigenvalues as function of doping of BSCCO and the degree of incommensuration of the spin fluctuations are shown in Fig. 1(b). Attractive eigenvalues $\left(\lambda_{\Gamma}<0\right)$ are found for the even-parity representations $A_{1 g, 2 g}, B_{1 g, 2 g}$, with the strongest attraction in the $B_{1 g}$ channel $\left(d_{x^{2}-y^{2}}\right.$ wave), followed by the $A_{2 g}$ channel (extended $s$ wave) for dominantly antiferromagnetic spin fluctuations. Also the odd-parity representation $E_{u}$ has attractive eigenvalues. Here it is the channel with $\boldsymbol{d} \| \hat{z}$ that is most attractive, because it is closest in value to that of the $B_{1 g}$ channel. This is parallel to what was found earlier in the case of $\mathrm{Sr}_{2} \mathrm{RuO}_{4} \cdot{ }^{28}$

Next, I need a theory to self-consistently solve for a vortex structure in the presence of a Zeeman field and competing order-parameter symmetries and use the quasiclassical theory, a leading-order theory in $\Delta / E_{F}(\ll 1)$, as presented in, e.g., Ref. 22. The central object is the Green's function $\hat{g}\left(\hat{\boldsymbol{p}}_{F}, \boldsymbol{R} ; \varepsilon\right)=(1 / a) \int d \xi_{p} \hat{\tau}_{3} \hat{G}(\boldsymbol{p}, \boldsymbol{R} ; \varepsilon)$, obeying the Eilenberger equation

$$
\begin{gathered}
i \boldsymbol{v}_{F} \cdot \nabla \hat{g}\left(\hat{\boldsymbol{p}}_{F}, \boldsymbol{R} ; \varepsilon\right)+\left[\varepsilon \hat{\tau}_{3}-\boldsymbol{h} \cdot \hat{\boldsymbol{\sigma}}-\hat{\Delta}\left(\hat{\boldsymbol{p}}_{F}, \boldsymbol{R}\right),\right. \\
\left.\hat{g}\left(\hat{\boldsymbol{p}}_{F}, \boldsymbol{R} ; \varepsilon\right)\right]=0,
\end{gathered}
$$

and the normalization condition $\hat{g}\left(\hat{\boldsymbol{p}}_{F}, \boldsymbol{R} ; \varepsilon\right)^{2}=-\pi^{2}$. The quasiclassical "Hamiltonian" $\varepsilon \hat{\tau}_{3}-\boldsymbol{h} \cdot \hat{\boldsymbol{\sigma}}-\hat{\Delta}\left(\hat{\boldsymbol{p}}_{F}, \boldsymbol{R}\right)$ is a $4 \times 4$ matrix in combined particle-hole $\left(\hat{\tau}_{i=1,2,3}\right)$ and spin space $\left(\sigma_{i=x, y, z}\right)$. The order-parameter matrix in (3) reads

$$
\hat{\Delta}\left(\hat{\boldsymbol{p}}_{F}, \boldsymbol{R}\right)=\left(\begin{array}{cc}
0 & \Delta\left(\hat{\boldsymbol{p}}_{F}, \boldsymbol{R}\right) \\
\tilde{\Delta}\left(\hat{\boldsymbol{p}}_{F}, \boldsymbol{R}\right) & 0
\end{array}\right),
$$

where $\Delta\left(\hat{\boldsymbol{p}}_{F}, \boldsymbol{R}\right)=\left[\Delta^{s}\left(\hat{\boldsymbol{p}}_{F}, \boldsymbol{R}\right)+\boldsymbol{\Delta}^{t}\left(\hat{\boldsymbol{p}}_{F}, \boldsymbol{R}\right) \cdot \boldsymbol{\sigma}\right] i \sigma_{y}$ is a $2 \times$ 2 spin-matrix order parameter. The superscripts refer to spin-singlet $(s)$ and spin-triplet $(t)$ components of the order parameter. Particle-hole components are related via the "tilde" symmetry $\tilde{\alpha}\left(\hat{\boldsymbol{p}}_{F}, \boldsymbol{R} ; \varepsilon, t\right)=$ $\alpha^{*}\left(-\hat{\boldsymbol{p}}_{F}, \boldsymbol{R} ;-\varepsilon^{*}, t\right)$, with * denoting complex conjugation. This gives $\tilde{\Delta}\left(\hat{\boldsymbol{p}}_{F}, \boldsymbol{R}\right)=i \sigma_{y}\left[\Delta^{s *}\left(-\hat{\boldsymbol{p}}_{F}, \boldsymbol{R}\right)-\boldsymbol{\Delta}^{t *}\left(-\hat{\boldsymbol{p}}_{F}, \boldsymbol{R}\right)\right.$. $\boldsymbol{\sigma}]=i \sigma_{y}\left[\Delta^{s *}\left(\hat{\boldsymbol{p}}_{F}, \boldsymbol{R}\right)+\boldsymbol{\Delta}^{t *}\left(\hat{\boldsymbol{p}}_{F}, \boldsymbol{R}\right) \cdot \boldsymbol{\sigma}\right]$. The Zeeman term in
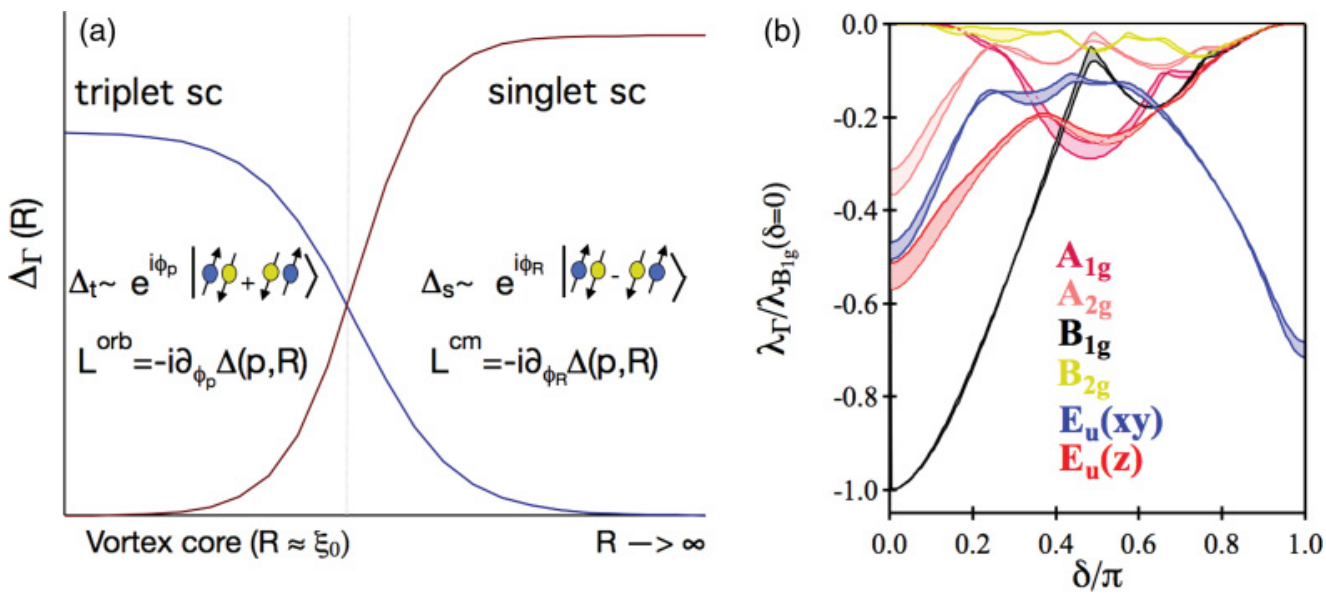

FIG. 1. (Color online) A schematic picture of the vortex-core state of a $d$-wave superconductor in the presence of a pairing attraction in a triplet channel is shown in (a). The core state $\left(R \lesssim \xi_{0}\right)$ may have a superconducting core with a sizable order-parameter amplitude with a $p_{x} \pm i p_{y}$ symmetry. (b) The coupling constants computed using a spin-fluctuation-mediated pairing interaction equation (1) and a simplified generic band structure of the cuprates. The parameter $\delta$ tunes from predominantly antiferromagnetic $(\delta=0)$ to ferromagnetic $(\delta=\pi)$ spin fluctuations. The width of the curves signifies the span from an overdoped $\left(\left|\lambda_{\Gamma}(\delta=0)\right|\right.$ smaller $)$ to an underdoped $\left(\left|\lambda_{\Gamma}(\delta=0)\right|\right.$ bigger $)$ material. Attraction in the spin-triplet pairing channel is sizable for all $\delta$. 

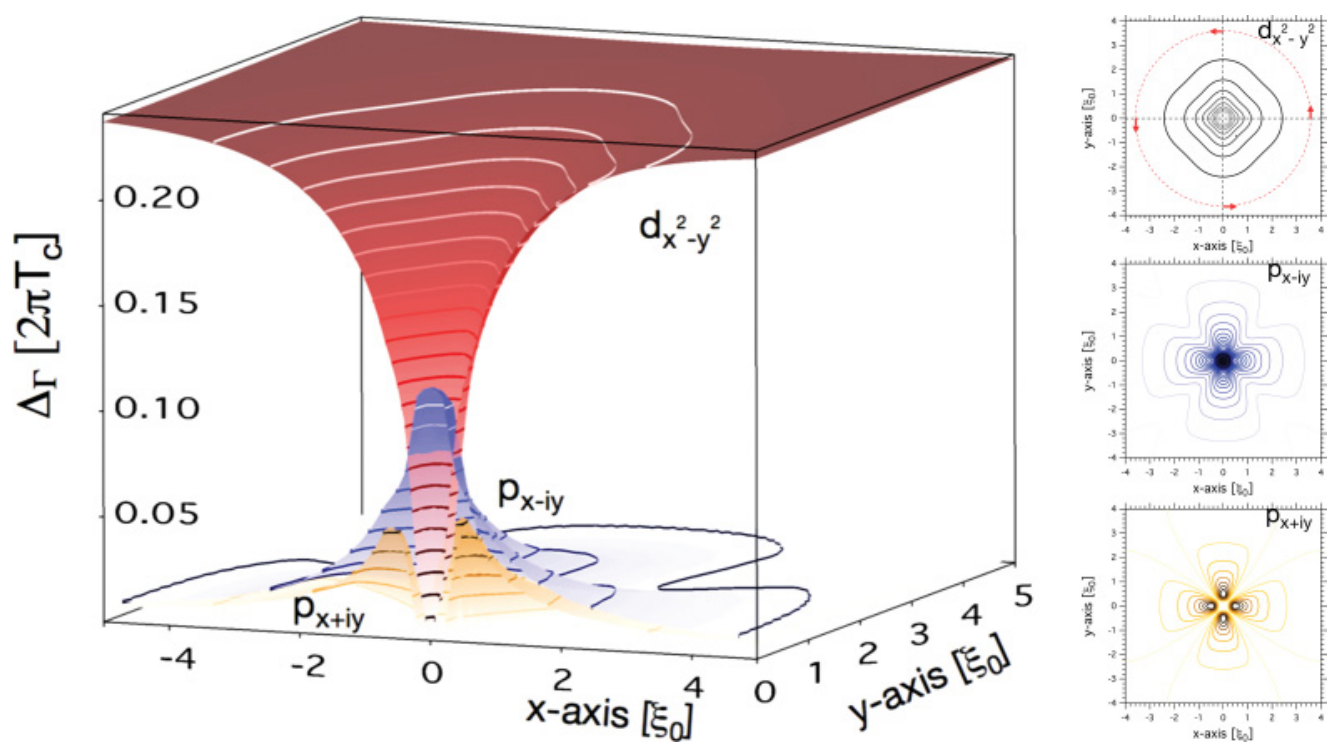

FIG. 2. (Color online) The order-parameter amplitudes computed at $T=0.05 T_{\mathrm{c}}$ with $\lambda_{E_{u}}=0.9 \lambda_{B_{1 g}}$ and $h=0.02 \Delta_{0}$, i.e., $2 \%$ of the zero-temperature $d$-wave gap $\Delta_{0}$. The asymptotic $d$-wave order parameter having a phase winding of $2 \pi$ is, as seen, suppressed in the core and heals to its bulk amplitude over roughly $5 \xi_{0}$ away from the core. In the vortex core a substantial triplet order parameter $\sim p_{x-i y}$ is stabilized, filling an area of $\sim \pi \xi_{0}^{2}$ with an amplitude close to $50 \%$ of the bulk value of the $d$-wave gap. This component has no phase winding but a relative phase of $-\pi / 2$ to the $d$-wave component. Away from the core a second component $\sim p_{x+i y}$ with a phase winding of $4 \pi$ appears.

(3) reads $\boldsymbol{h} \cdot \hat{\boldsymbol{\sigma}}=\operatorname{diag}\left[\boldsymbol{h} \cdot \boldsymbol{\sigma},-\sigma_{y} \boldsymbol{h} \cdot \boldsymbol{\sigma} \sigma_{y}\right]$, with the Zeeman field pinning the spin-quantization axis to the $z$ axis, $\boldsymbol{h} \cdot \boldsymbol{\sigma}=$ $-\mu_{B} B \sigma_{z} . \boldsymbol{h}$ is assumed to be homogeneous and small, $|\boldsymbol{h}|=$ $h \ll\left|\Delta_{0}\right|$, to weakly break parity ( $\Delta_{0}$ is the zero-temperature $d$-wave gap).

The Eilenberger equation (3) may be solved by introducing the following two spin-matrix coherence functions $\gamma=\left(\gamma_{s}+\boldsymbol{\gamma}_{t} \cdot \boldsymbol{\sigma}\right) i \sigma_{y}$ and $\tilde{\gamma}=i \sigma_{y}\left(\tilde{\gamma}_{s}-\tilde{\boldsymbol{\gamma}}_{t} \cdot \boldsymbol{\sigma}\right)$ parametrizing the retarded quasiclassical Green's function ${ }^{10,22,30}$

$$
\hat{g}^{R}=-i \pi \hat{N}\left(\begin{array}{cc}
1+\gamma \tilde{\gamma} & 2 \gamma \\
-2 \tilde{\gamma} & -1-\tilde{\gamma} \gamma
\end{array}\right)=\left(\begin{array}{cc}
g & f \\
\tilde{f} & \tilde{g}
\end{array}\right),
$$

with $\hat{N}=\operatorname{diag}\left[(1-\gamma \tilde{\gamma})^{-1},(1-\tilde{\gamma} \gamma)^{-1}\right]$. The advanced function is given as $\hat{g}^{A}=\tau_{3} \hat{g}^{R \dagger} \tau_{3}$ and the Matsubara function as $\hat{g}^{M}\left(\epsilon_{n}\right)=\hat{g}^{R}\left(\epsilon+i 0 \rightarrow i \epsilon_{n}\right)$. The mixed-parity order parameter components are linear combinations of the singlet and the $z$ component of the triplet part as $\Delta_{ \pm}\left(\hat{\boldsymbol{p}}_{F}, \boldsymbol{R}\right)= \pm\left[\Delta^{s}\left(\hat{\boldsymbol{p}}_{F}, \boldsymbol{R}\right) \pm \Delta_{z}^{t}\left(\hat{\boldsymbol{p}}_{F}, \boldsymbol{R}\right)\right]$ [and $\tilde{\Delta}_{ \pm}\left(\hat{\boldsymbol{p}}_{F}, \boldsymbol{R}\right)=$ $\left.\pm\left[\Delta^{s *}\left(\hat{\boldsymbol{p}}_{F}, \boldsymbol{R}\right) \pm \Delta_{z}^{t *}\left(\hat{\boldsymbol{p}}_{F}, \boldsymbol{R}\right)\right]\right]$. This leads, together with the Zeeman-shifted frequency $\varepsilon_{ \pm}=\varepsilon \pm \mu_{B} B$, to a separation in to two pseudospin bands $( \pm)$ with different order parameters $\Delta_{ \pm}\left(\hat{\boldsymbol{p}}_{F}, \boldsymbol{R}\right)$. and Eq. (3) is written as two pairs of scalar Riccati equations

$$
\begin{aligned}
& i \boldsymbol{v}_{F} \cdot \nabla \gamma_{ \pm}+2 \varepsilon_{ \pm} \gamma_{ \pm}=-\gamma_{ \pm} \tilde{\Delta}_{ \pm} \gamma_{ \pm}-\Delta_{ \pm}, \\
& i \boldsymbol{v}_{F} \cdot \nabla \tilde{\gamma}_{ \pm}-2 \varepsilon_{ \pm} \tilde{\gamma}_{ \pm}=-\tilde{\gamma}_{ \pm} \Delta_{ \pm} \tilde{\gamma}_{ \pm}-\tilde{\Delta}_{ \pm},
\end{aligned}
$$

one for each spin band. The two equations are solved by numerical integration along straight lines, or trajectories, $\boldsymbol{s}(x)=\boldsymbol{s}_{0} \pm x \boldsymbol{v}_{F} /\left|\boldsymbol{v}_{F}\right|$ for $\gamma_{ \pm}$and $\tilde{\gamma}_{ \pm}$as described in, e.g., Ref. 22. Once $\gamma_{ \pm}\left(\hat{\boldsymbol{p}}_{F}, \boldsymbol{R} ; \epsilon\right)$ and $\tilde{\gamma}_{ \pm}\left(\hat{\boldsymbol{p}}_{F}, \boldsymbol{R} ; \epsilon\right)$ are obtained, the order-parameter fields are calculated using (2) as

$$
\begin{array}{r}
\Delta_{d}^{s}(\boldsymbol{R})=-\lambda_{B_{1 g}} T \sum_{\left|\epsilon_{n}\right| \leqslant \epsilon_{c}}\left\langle\mathcal{Y}_{B_{1 g}}^{*}\left(\hat{\boldsymbol{p}}_{F}\right) \frac{\left(f_{+}-f_{-}\right)}{2}\right\rangle_{\hat{\boldsymbol{p}}_{F}}, \\
\Delta_{p_{x} \pm i p_{y}}^{t}(\boldsymbol{R})=-\lambda_{E_{u}} T \sum_{\left|\epsilon_{n}\right| \leqslant \epsilon_{c}}\left\langle\mathcal{Y}_{E_{u}, \pm}^{*}\left(\hat{\boldsymbol{p}}_{F}\right) \frac{\left(f_{+}+f_{-}\right)}{2}\right\rangle_{\hat{\boldsymbol{p}}_{F}},
\end{array}
$$

where $f_{ \pm}=f_{ \pm}\left(\hat{\boldsymbol{p}}_{F}, \boldsymbol{R} ; \epsilon_{n}\right)$ are the anomalous functions on spin-band $\pm .\langle\cdots\rangle_{\hat{\boldsymbol{p}}_{F}}=\int \frac{d \phi_{p}}{2 \pi}$ is the average over the momentum direction $\hat{\boldsymbol{p}}_{F}$ on the Fermi surface, with the angle $\phi_{p}$ giving the angle the momentum $\hat{\boldsymbol{p}}_{F}$ makes to the crystal $a$ axis ( $x$ axis in the figures). In the vortex calculations the conventional basis functions $\mathcal{Y}_{B_{1 g}}\left(\hat{\boldsymbol{p}}_{F}\right)=\sqrt{2} \cos 2 \phi_{p}$ and $\mathcal{Y}_{E_{u}, \pm}\left(\hat{\boldsymbol{p}}_{F}\right)=\sqrt{2}\left(\cos \phi_{p} \pm i \sin \phi_{p}\right)$ are used. The pairing interaction and cutoff frequency $\epsilon_{c}$ are eliminated in favor of the transition temperature $T_{\mathrm{c}}$ as $-\lambda_{B_{1 g}}^{-1}=\ln T / T_{\mathrm{c}}+\sum_{n \geqslant 0}^{n \leqslant n_{\mathrm{c}}}(n+$ $1 / 2)^{-1}$. The subdominant interaction $\lambda_{E_{u}}$ is introduced in the self-consistent calculations as a fraction of the dominant one and is treated as a parameter that is free to explore.

\section{RESULTS}

Equations (6)-(9) are iterated until self-consistency is reached. In Fig. 2, the structure of a mixed-parity vortex is shown. A substantial triplet $p$-wave order parameter may be nucleated in the singlet $d$-wave vortex core with both $p$-wave components $p_{x} \pm i p_{y}$ present. The $p$-wave component $\Delta_{p}$ with $L_{z, p \text {-wave }}^{\text {orb }}=L_{z, d \text {-wave }}^{\text {c.mill be finite in the core center and }}$ it carries no phase winding. The $p$-wave component $\Delta_{p^{\prime}}$ with $L_{z, p^{\prime} \text {-wave }}^{\text {orb }}=-L_{z, d \text {-wave }}^{\text {c.m. }}$ has a finite amplitude on the phase boundary separating the singlet and triplet order parameters, at $R \sim \xi_{0}=\hbar v_{F} / 2 \pi T_{\mathrm{c}}$, around which its phase winds by $4 \pi$. This is so that $L_{z, p^{\prime} \text {-wave }}^{\text {orb }}+L_{z, p^{\prime} \text {-wave }}^{\text {c.m. }}=L_{d \text {-wave }}^{\text {c.m. }}$. All amplitudes 


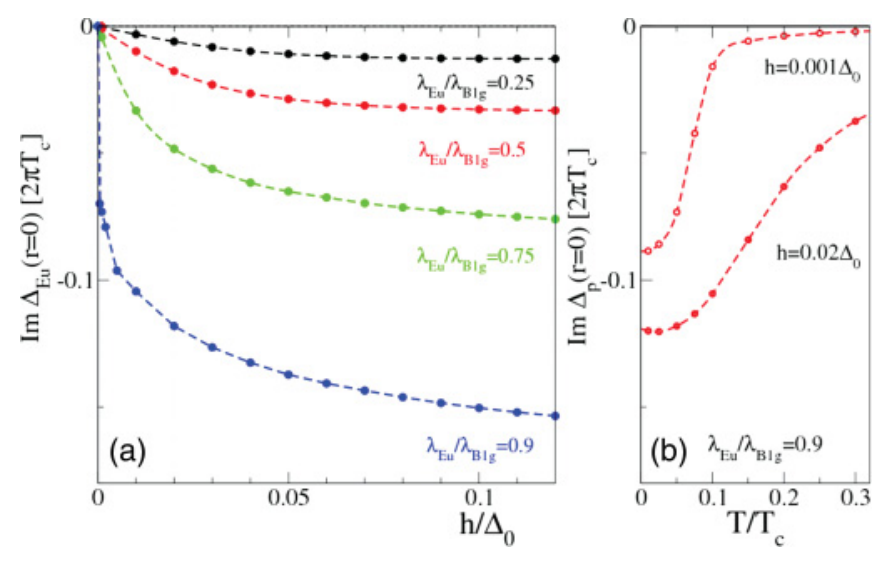

FIG. 3. (Color online) The amplitude of the $p$-wave order parameter in the vortex core $\Delta_{p}(0)$, computed at $T=0.05 T_{\mathrm{c}}$, is shown in (a) as a function of the symmetry-breaking field $h$ for various ratios $\lambda_{E_{u}} / \lambda_{B_{1 g}}$. For large ratios $\lambda_{E_{u}} / \lambda_{B_{1 g}} \lesssim 0.9$, the $p$ wave stabilizes even as $h \rightarrow 0$ (but $h$ finite). The temperature dependence of $\Delta_{p}(0)$ is shown in (b) at $h=0.001 \Delta_{0}$ and at $h=0.02 \Delta_{0}$. For the larger field $\Delta_{p}(0 ; T)$ is finite at higher temperatures and grows with decreasing temperature, while for $h=0.001 \Delta_{0}, \Delta_{p}(0 ; T)$ has a distinct temperature below which it grows rapidly to its low- $T$ value. For smaller ratios $\lambda_{E_{u}} / \lambda_{B_{1 g}} \lesssim 0.7$, the $p$-wave core phase is close to linearly dependent on $h$.

retain the fourfold symmetry of the $d_{x^{2}-y^{2}}$ amplitude, as seen in the contour plots displayed in Fig. 2.
The nucleation of a $p$-wave order parameter is dependent on a finite Zeeman field. In Fig. 3 the amplitude $\Delta_{p}(0)$ is displayed as a function of $h$ for different strengths of $\lambda_{E_{u}} . \Delta_{p}(0)$ is finite for all coupling strengths and grows with increasing Zeeman field. For larger couplings $\lambda_{E_{u}} \gtrsim 0.7 \lambda_{B_{1 g}}$, the onset of $\Delta_{p}(0)$ at small fields becomes increasingly nonlinear, with a sharp onset of the sub-dominant order parameter at $h \gtrsim 0$. In Fig. 3(b) the temperature dependence of $\Delta_{p}(0)$ is shown. While the $p$-wave amplitude is finite at all temperatures, there is a transition from a field-induced triplet order parameter at larger fields, when $h \gtrsim 0.02 \Delta_{0}$, and at high temperatures $T \gtrsim 0.1 T_{\mathrm{c}}$, to an intrinsic phase transition at vanishingly small fields $h \lesssim 0.01 \Delta_{0}$ and low temperatures. For weaker triplet pairing strength the intrinsic phase transition into a $d+i p$ vortex state is pushed to lower temperatures.

The emergence of a mixed-parity state in the vortex center at low temperatures has a profound effect on the quasiparticle spectra. In general, the spatially resolved density of states (DOS) $N_{\text {Tot }}(\boldsymbol{R} ; \epsilon)=-\frac{1}{\pi} \operatorname{Im}\left\langle\operatorname{Tr}\left[\hat{\tau}_{3} \hat{g}^{R}\left(\hat{\boldsymbol{p}}_{F}, \boldsymbol{R} ; \epsilon+\right.\right.\right.$ $\left.\left.\left.i 0^{+}\right)\right]\right\rangle_{\hat{\boldsymbol{p}}_{F}}$ will show evidence of the subgap Caroli-de GennesMatricon states that carry the screening current of a vortex. ${ }^{1,31}$ Resolving the spectra also in position on the Fermi surface $\hat{\boldsymbol{p}}_{F}$, one finds for a pure $d$-wave vortex, on trajectories tangential to the asymptotic phase winding, that the bound states in the core have a qualitative dispersion with a distance or "impact parameter" $b$ from the vortex core $E\left(\hat{\boldsymbol{p}}_{F}, b\right) \approx \pm\left|\Delta\left(\hat{\boldsymbol{p}}_{F}\right)\right| \tanh \left(b / b_{0}\right)$. Here, $b_{0} \approx \xi_{0}$ is a scale factor and \pm denotes if the momentum
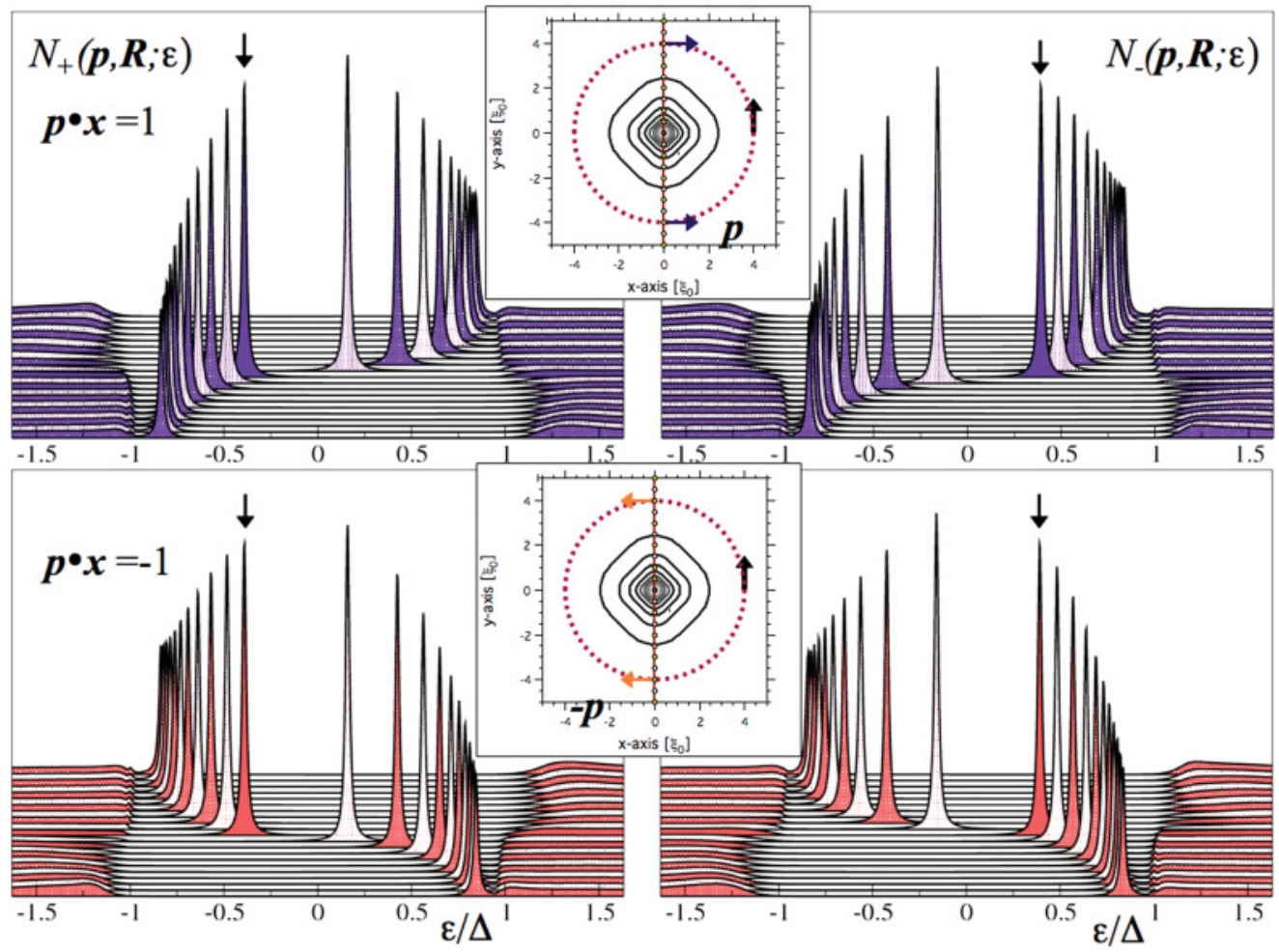

FIG. 4. (Color online) The trajectory-resolved DOS $N_{ \pm}\left(\hat{\boldsymbol{p}}_{F}, \boldsymbol{R} ; \epsilon\right)$ evaluated on the cut along the $y$ axis through the origin. The spectra are taken in the indicated points separated by $0.5 \xi_{0}$ (see the inserts). The phase winding of the $d$ wave is counterclockwise in this case. The direction of $\hat{\boldsymbol{p}}_{F}$ is chosen to be (anti)parallel to the $x$ axis in (lower) upper pair of panels. The presence of a subdominant order-parameter amplitude introduces a shift of the the state in the core center to a finite energy (this state is marked by an arrow in each panel). This energy shift is negative (positive) for the branch $N_{+}\left(N_{-}\right)$. There still exist zero-energy states on both branches, but these are now found away from the core center. 

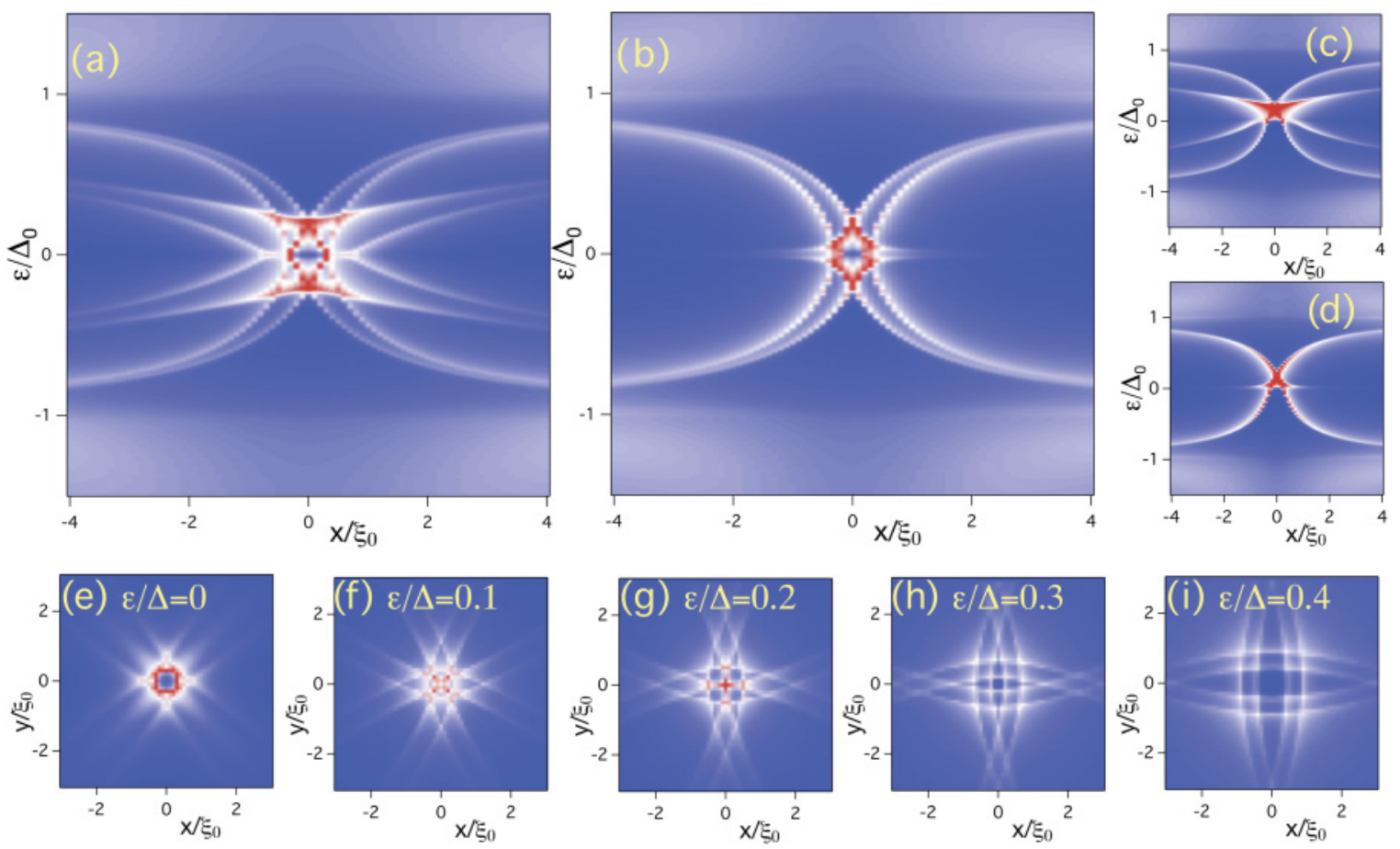

FIG. 5. (Color online) The DOS of a vortex with a finite $p$-wave core phase. In (a) and (b) $N_{\text {Tot }}(\boldsymbol{R} ; \epsilon)$ is displayed as a function of distance from the core in (a) along the $y$ axis at $x=0$ and in (b) along $x=y$. In (c) and (d), as in (a) and (b), the DOS $N_{-}(\boldsymbol{R} ; \epsilon)$ for one spin band. There is a dispersion of the core states as a function of distance from the core, but as seen in the spin-resolved DOS, the total DOS also consists of two nondispersing states with a large spectral weight located at $\epsilon= \pm 0.2 \Delta_{0}$. In panels (e)-(i) a spatial map of the DOS is shown at various $\epsilon$.

direction is parallel $(+)$ or antiparallel $(-)$ to the phase winding of the asymptotic order parameter $\Delta\left(\hat{\boldsymbol{p}}_{F}\right) e^{i \phi}$ at $b \ll 0$. Introducing an imaginary $p$-wave order parameter in the core splits the quasiparticle spectra into two branches, one for each spin band $\alpha(= \pm)$. This is displayed in Fig. 4. The qualitative quasiparticle dispersion of the core states is now modified as $E_{\alpha}\left(\hat{\boldsymbol{p}}_{F}, b\right) \approx \pm\left|\Delta\left(\hat{\boldsymbol{p}}_{F}\right)\right| \tanh \left[\left(b \mp s_{\alpha} \bar{b}\right) / b_{0}\right]$, where $s_{ \pm}= \pm 1$. The offset $\bar{b}$ is a direct consequence of a finite order parameter in the vortex-core center and shifts the zero-energy state to a finite impact parameter away from the core center. In the core center the quasiparticle state is shifted to finite energy $E_{\alpha}\left(\hat{\boldsymbol{p}}_{F}, 0\right) \approx-s_{\alpha}\left|\Delta\left(\hat{\boldsymbol{p}}_{F}\right)\right| \tanh \left(\bar{b} / b_{0}\right)$. Note that both states, with and against the phase winding $( \pm)$ on one branch, are shifted to the same energy. This leads to a suppression of the screening current in the core area $|\boldsymbol{R}| \lesssim 1 \xi_{0}$.

The features of the trajectory-resolved DOS are detectable in the trajectory-averaged total DOS, $N_{\text {Tot }}(\boldsymbol{R} ; \epsilon)$, which is directly related to the tunneling conductance measured by STS. ${ }^{8}$ In Fig. 5 the DOS calculated at $T=0.025 T_{\text {c }}$ with $\lambda_{E_{u}}=0.9 \lambda_{B_{1 g}}$ and $h=0.02 \Delta_{0}$ is shown. $N_{\text {Tot }}(\boldsymbol{R} ; \epsilon)$ on a ray through the vortex core along an antinode [Fig. 5(a)] lacks a zero energy state (ZES) in the core center. The core state is pushed to $E_{\alpha}(0) \approx \mp 0.3 \Delta_{0}$, and this state has very little dispersion with its position on the ray. ZESs are found at a distance $b \approx \pm 0.5 \xi_{0}$ from the core center with half the spectral weight of the ZES in a pure $d$-wave vortex as the two spin bands are shifted differently by $\Delta_{p}(0)$. The features in the DOS are also generally broadened by the angle average $\langle\cdots\rangle_{\hat{\boldsymbol{p}}_{F}}$. The corresponding spin-band-resolved DOS is shown in Fig. 5(c) for spin band $(-)$. On a ray through the vortex core along a node [Fig. 5(b)] the DOS also lack ZESs in the core center, but the core state $E_{\alpha}(0) \approx \mp 0.3 \Delta_{0}$ has more of a dispersion with small $b$ as compared to that in the antinodal direction. This is due to the linear opening of the energy gap around the node $\left|\Delta_{d}(\phi)\right| \sim\left|(\partial \Delta / \partial \phi)\left(\phi-\phi_{\text {node }}\right)\right|$ of the $d$-wave gap. In Figs. 5(e)-5(i) the spatially resolved DOS is displayed on a $6 \xi_{0} \times 6 \xi_{0}$ square with the center in the vortex core at different fixed energies. The ZESs form a ring around the core center and the cores states, $E_{\alpha}(0) \approx \mp 0.3 \Delta_{0}$, extend along the antinodes. The overall shape of the vortex is a doubling of the star-shaped DOS found in the pure $d$-wave vortex core. ${ }^{10}$ The doubling shows up as a square lattice in the DOS amplitude, and the lattice constant is set by the magnitude of the induced triplet order parameter $\Delta_{p}(0)$.

\section{CONCLUSIONS}

In closing, I have shown that a mixed-parity $d+i p$ vortex state is possible to stabilize in a high- $T_{\mathrm{c}}$ superconductor. The weak Zeeman coupling to the external magnetic field gives a sufficient seed to nucleate a $p$-wave order parameter in the vortex core. The necessary attractive triplet pairing channel is supported by spin-fluctuation-mediated pairing, argued to be relevant for the cuprates. This unique core state is directly detectable in STS measurements of the quasiparticle spectra, and I find, within the limits of quasiclassical theory, good agreement with existing experimental data. ${ }^{4-8}$ A further study using the Bogoliubov-de Gennes equations is needed to quantitatively compare the theory with experiments. This is because the quasiclassical theory does not self-consistently resolve the angular-momentum quantization of the Caroli-de 
Gennes-Matricon states. This quantization gives a finite shift, or a minigap, of the lowest-energy state from the Fermi surface $\sim \Delta^{2} / E_{F} .1,3$ For the high- $T_{\text {c }}$ cuprates this minigap may be sizable as $\Delta / E_{F} \approx 0.1$ and may very well remove the states found at $E=0.0$ away from the core center.

\section{ACKNOWLEDGMENT}

Interaction with Øystein Fischer during this work, from the initial curious questions to discussing results, has been extremely valuable.
${ }^{1}$ C. Caroli, P. G. de Gennes, and J. Matricon, Phys. Lett. 9, 307 (1964).

${ }^{2}$ H. F. Hess, R. B. Robinson, R. C. Dynes, J. M. Valles Jr., and J. V. Waszczak, Phys. Rev. Lett. 62, 214 (1989).

${ }^{3}$ F. Gygi and M. Schlüter, Phys. Rev. B 43, 7609 (1991).

${ }^{4}$ I. Maggio-Aprile, C. Renner, A. Erb, E. Walker, and Ø. Fischer, Phys. Rev. Lett. 75, 2754 (1995).

${ }^{5}$ S. H. Pan, E. W. Hudson, A. K. Gupta, K. W. Ng, H. Eisaki, S. Uchida, and J. C. Davis, Phys. Rev. Lett. 85, 1536 (2000).

${ }^{6}$ J. E. Hoffman, E. W. Hudson, K. M. Lang, V. Madhavan, H. Eisaki, S. Uchida, and J. C. Davis, Science 295, 466 (2002).

${ }^{7}$ G. Levy, M. Kugler, A. A. Manuel, Ø. Fischer, and M. Li, Phys. Rev. Lett. 95, 257005 (2005).

${ }^{8} \varnothing$. Fischer, M. Kugler, I. Maggio-Aprile, C. Berthod, and C. Renner, Rev. Mod. Phys. 79, 353 (2007).

${ }^{9}$ Y. Wang and A. H. MacDonald, Phys. Rev. B 52, 3876 (1995).

${ }^{10}$ N. Schopohl and K. Maki, Phys. Rev. B 52, 490 (1995).

${ }^{11}$ M. Ichioka, N. Enomoto, N. Hayashi, and K. Machida, Phys. Rev. B 53, 2233 (1996).

${ }^{12}$ M. Ichioka, N. Hayashi, N. Enomoto, and K. Machida, Phys. Rev. B 53, 15316 (1996).

${ }^{13}$ M. Franz and Z. Tesanovic, Phys. Rev. Lett. 80, 4763 (1998).

${ }^{14}$ D. P. Arovas, A. J. Berlinsky, C. Kallin, and Shou-Cheng Zhang, Phys. Rev. Lett. 79, 2871 (1997).
${ }^{15}$ B. M. Andersen, H. Bruus, and P. Hedegård, Phys. Rev. B 61, 6298 (2000).

${ }^{16}$ C. Berthod and B. Giovannini, Phys. Rev. Lett. 87, 277002 (2001).

${ }^{17}$ M. M. Maśka and M. Mierzejewski, Phys. Rev. B 68, 024513 (2003).

${ }^{18}$ M. M. Salomaa and G. E. Volovik, Rev. Mod. Phys. 59, 533 (1987).

${ }^{19}$ A. G. Lebed, Phys. Rev. Lett. 96, 037002 (2006).

${ }^{20}$ V. V. Kabanov, Phys. Rev. B 76, 172501 (2007).

${ }^{21}$ O. Dutta and A. G. Lebed, Phys. Rev. B 78, 224504 (2008).

${ }^{22}$ J. A. Sauls and M. Eschrig, New J. Phys. 11, 075008 (2009).

${ }^{23}$ P. Hakonen, O. V. Lounasmaa, and J. Simola, Physica B 160, 1 (1989).

${ }^{24}$ M. M. Salomaa and G. E. Volovik, Phys. Rev. Lett. 51, 2040 (1983).

${ }^{25}$ E. V. Thuneberg, Phys. Rev. B 36, 3583 (1987).

${ }^{26}$ M. Fogelström and J. Kurkijärvi, J. Low Temp. Phys. 98, 195 (1995).

${ }^{27}$ M. Eschrig, Adv. Phys. 55, 47 (2006).

${ }^{28}$ M. Eschrig, J. Ferrer, and M. Fogelström, Phys. Rev. B 63, 220509 (2001).

${ }^{29}$ B. W. Hoogenboom, C. Berthod, M. Peter, $\varnothing$. Fischer, and A. A. Kordyuk, Phys. Rev. B 67, 224502 (2003).

${ }^{30}$ Y. Nagato, K. Nagai, and J. Hara, J. Low Temp. Phys. 93, 33 (1993).

${ }^{31}$ D. Rainer, J. A. Sauls, and D. Waxman, Phys. Rev. B 54, 10094 (1996). 